\title{
Cyclic Relatively Nonexpansive Mappings with Respect to Orbits and Best Proximity Point Theorems
}

\author{
Laishram Shanjit $\mathbb{D}^{1}{ }^{1}$ Yumnam Rohen, ${ }^{1}$ and K. Anthony Singh ${ }^{2}$ \\ ${ }^{1}$ Department of Mathematics, National Institute of Technology Manipur, Langol 795004, India \\ ${ }^{2}$ Department of Mathematics, D. M. College of Science, Imphal, Manipur 795001, India
}

Correspondence should be addressed to Laishram Shanjit; shanjit2016@nitmanipur.ac.in

Received 25 November 2020; Revised 26 December 2020; Accepted 6 January 2021; Published 8 February 2021

Academic Editor: Ali Jaballah

Copyright (C) 2021 Laishram Shanjit et al. This is an open access article distributed under the Creative Commons Attribution License, which permits unrestricted use, distribution, and reproduction in any medium, provided the original work is properly cited.

In this article, we introduce cyclic relatively nonexpansive mappings with respect to orbits and prove that every cyclic relatively nonexpansive mapping with respect to orbits $T$ satisfying $T(A) \subseteq B, T(B) \subseteq A$ has a best proximity point. We also prove that Mann's iteration process for a noncyclic relatively nonexpansive mapping with respect to orbits converges to a fixed point. These relatively nonexpansive mappings with respect to orbits need not be continuous. Some illustrations are given in support of our results.

\section{Introduction and Preliminaries}

In the year 1948, Brodskil et al. [1] introduced a geometrical property known as the normal structure to study the existence of a common fixed point for a family of isometry mappings. In [2], Kirk used a geometrical notion, namely, normal structure for a nonempty bounded closed and convex subset $K$ of a reflexive Banach space $X$, thereby getting the existence of a fixed point for a mapping that does not increase distance (a nonexpansive mapping). Let $(G, H)$ be a pair of subsets in a Banach space $X$. A mapping $T: G \cup H \longrightarrow G \cup H$ is said to be a relatively nonexpansive mapping if $T(G) \subseteq H, T(H) \subseteq G$ (or $T(G) \subseteq G, T(H) \subseteq H)$ and $\quad\|T u-T v\| \leq\|u-v\|$, for $u \in G, v \in H$. If $G=H$, then $T$ is a nonexpansive self-mapping. In the year 2005, Eldred et al. [3] introduced a geometrical notion called proximal normal structure, for a nonempty, convex weakly compact pair $(G, H)$, in order to study the existence of a fixed point (the best proximity point) for relatively nonexpansive mappings under suitable assumptions. Motivated by Harandi et al. [4], in the year 2020, Gabeleh et al. [5] introduced a notion called noncyclic relatively nonexpansive mapping with respect to orbits $T$ satisfying $T(G) \subseteq G, T(H) \subseteq H$ and showed the existence of a fixed point (best proximity point) under certain assumptions. In the setting of Banach spaces, the existence of fixed point and best proximity point theorems, respectively, for nonexpansive and relatively nonexpansive mappings has been extensively studied by many authors (see $[3,5-8])$. For more exciting results about the best proximity point for contraction and multivalued mappings studied by many authors, see [9-12]. For any pair of subsets $(C, D)$ of $X$, the following notations will be used in the sequel:

$$
\begin{aligned}
R(u, D) & :=\sup \{\|u-v\|: v \in D\}, \quad u \in C ; \\
\delta(C, D) & :=\sup \{R(u, D): u \in C\} ; \\
C_{0} & :=\{u \in C:\|u-v\|=\operatorname{dist}(C, D), v \in D\} ; \\
D_{0} & :=\{v \in D:\|u-v\|=\operatorname{dist}(C, D), u \in C\},
\end{aligned}
$$

where $\operatorname{dist}(C, D):=\inf \{\|u-v\|: u \in C, v \in D\}$.

Definition 1 (see [3]). A nonempty pair $(C, D)$ of subsets in a normed linear space $X$ is known as a proximal pair if for every $u_{1} \in C, v_{1} \in D$ there exist $u_{2} \in C, v_{2} \in D$ such that $\left\|u_{1}-v_{2}\right\|=\operatorname{dist}(C, D)=\left\|u_{2}-v_{1}\right\|$.

Note: the pair $\left(C_{0}, D_{0}\right)$ is a proximal pair and $\left(C_{0}, D_{0}\right) \subseteq$ $(C, D)$, that is $C_{0} \subseteq C, D_{0} \subseteq D$.

Definition 2 (see [13]). A nonempty pair $(C, D)$ of subsets in a Banach space $X$ is said to be a proximal parallel pair if 
(i) $(C, D)$ is a sharp proximal pair, that is, for each $\left(u_{1}, v_{1}\right) \in C \times D$, there is unique element $\left(u_{2}, v_{2}\right) \in C \times D \quad$ such that $\left\|u_{1}-v_{2}\right\|=\operatorname{dist}(C, D)=\left\|u_{2}-v_{1}\right\|$.

(ii) $D=C+h$, for a certain $h \in X$.

By using the above definition, Rajesh et al. [14] introduced the rectangle property as follows.

Definition 3 Let $X$ be a Banach space and let $(C, D)$ be a nonempty, convex proximal parallel pair. The nonempty proximal parallel pair $(C, D)$ is assumed to have the rectangle property iff $\|u+h-v\|=\|v+h-u\|$, for $u, v \in C$, where $D=C+h, h \in X$.

Definition 4 (see [3]). Let $X$ be a Banach space. A nonempty convex pair $(G, H)$ of subsets in $X$ is assumed to have proximal normal structure if for every nonempty, closed convex bounded proximal pair $(C, D) \subseteq(G, H)$ for which $\operatorname{dist}(C, D)=\operatorname{dist}(G, H)$ and $\delta(C, D)>\operatorname{dist}(C, D)$, there is $(u, v) \in(C, D)$ such that

$$
\max \{R(u, D), R(v, C)\}<\delta(C, D) .
$$

The following two theorems are obtained from Eldred et al. [3].

Theorem 1. Let $X$ be a Banach space and let $(G, H)$ be a nonempty, convex weakly compact proximal pair in $X$. Let $T: G \cup H \longrightarrow G \cup H \quad$ with $T(G) \subseteq H, T(H) \subseteq G$ and $\|T u-T v\| \leq\|u-v\|$, for $u \in G, v \in H$. Suppose $(G, H)$ has proximal normal structure. Then, there exist $u_{0} \in G, v_{0} \in H$ such that $\left\|u_{0}-T u_{0}\right\|=\operatorname{dist}(G, H)=\left\|v_{0}-T v_{0}\right\|$.

Theorem 2. Let $X$ be a strictly convex Banach space and let $(G, H)$ be a nonempty, convex weakly compact proximal pair in $X$. Let $T: G \cup H \longrightarrow G \cup H$ with $T(G) \subseteq G, T(H) \subseteq H$ and $\|T u-T v\| \leq\|u-v\|$, for $u \in G, v \in H$. Suppose $(G, H)$ has proximal normal structure. Then, there exist $u_{0} \in G, v_{0} \in H$ such that $T u_{0}=u_{0}, T v_{0}=v_{0}$ and $\left\|u_{0}-v_{0}\right\|=\operatorname{dist}(G, H)$.

Remark 1. In the above two theorems, if $G=H$, then the main result of Kirk [2] is obtained.

Definition 5 (see [15]). A uniformly convex space $X$ is a normed linear space such that for each $\varepsilon>0$,

$$
\inf \left\{1-\frac{\|u+v\|}{2}:\|u\| \leq 1,\|v\| \leq 1,\|u-v\| \geq \varepsilon\right\}>0 .
$$

Moreover, if $u, v, w \in X, R>0,0 \leq \varepsilon \leq 2 R$, such that $\|u-w\| \leq R,\|v-w\| \leq R,\|u-v\| \geq \varepsilon$ which implies

$$
\left\|\frac{u+v}{2}-w\right\| \leq\left(1-\delta\left(\frac{\varepsilon}{R}\right)\right) R .
$$

The following result based on Mann's iteration process is well known.
Proposition 1 (see [16]). Let $X$ be a uniformly convex space, $\varepsilon>0$, and $0<\xi<1$. For any $R>0$, if $u, v \in X,\|u\| \leq R,\|v\| \leq R$, $\|u-v\| \geq \varepsilon$, then there is $\delta=\delta(\varepsilon / R)>0$, so that

$$
\|\xi u+(1-\xi) v\| \leq\left(1-2 \delta\left(\frac{\varepsilon}{R}\right) \min \{\xi, 1-\xi\}\right) R .
$$

Espinola proved the following result.

Lemma 1 (see [13]). Let $X$ be a strictly convex Banach space. If $(C, D)$ is a proximal pair in $X$, then $(C, D)$ is a proximal parallel pair.

Definition 6 (see [5]). Let $X$ be a Banach space and let $G$ and $H$ be nonempty subsets of $X$. A mapping $T: G \cup H \longrightarrow G \cup H$ satisfying $T(G) \subseteq G, T(H) \subseteq H$ is known as noncyclic relatively nonexpansive mapping with respect to orbits provided that for every $(u, v) \in G \times H$ if $\|u-v\|=\operatorname{dist}(G, H), \quad$ then $\quad\|T u-T v\|=\operatorname{dist}(G, H)$; otherwise,

$$
\|T u-T v\| \leq R(u, O(v)),\|T u-T v\| \leq R(v, O(u)),
$$

where $O(v):=\left\{v, T v, \ldots, T^{n} v, \ldots\right\} \subseteq H$ and $O(u) \subseteq G$.

In the above definition, if $G=H$, we obtain the definition of Harandi et al. [4] as follows.

Definition 7. Let $X$ be a Banach space and $H$ be a nonempty subset of $X$. A mapping $T: H \longrightarrow H$ is known as a nonexpansive mapping with respect to orbits if

$$
\|T u-T v\| \leq R(u, O(v)), \quad \forall u, v \in H .
$$

Remark 2. Every nonexpansive mapping is a nonexpansive mapping with respect to orbits, whereas the converse may not be true.

\section{Results for Cyclic Relatively Nonexpansive Mappings with respect to Orbits}

Motivated by Harandi et al. [4] and Gabeleh et al. [5], we define the following definition which will be extensively used in the sequel.

Definition 8. Let $G$ and $H$ be nonempty subsets of a Banach space $X$. A mapping $T: G \cup H \longrightarrow G \cup H$ satisfying $T(G) \subseteq H, T(H) \subseteq G$ is known as cyclic relatively nonexpansive mapping with respect to orbits provided that for every $(u, v) \in G \times H \quad$ if $\quad\|u-v\|=\operatorname{dist}(G, H)$, then $\|T u-T v\|=\operatorname{dist}(G, H)$; otherwise,

$$
\|T u-T v\| \leq R(u, O(v)),\|T v-T u\| \leq R(v, O(u)),
$$

where $O(v):=\left\{v, T v, \ldots, T^{n} v, \ldots\right\}, T^{2 n+1} v \in G, T^{2 n} v \in H$, and $O(u) \subseteq G \cup H$ for $n=0,1,2, \ldots$.

Example 1. Let $X=\ell^{\infty}\left(=\ell^{\infty}(\mathbb{N})\right)$ and let $\left\{e_{m}\right\}=\left\{\delta_{m}^{k}\right\}$ be the standard basis for $\ell^{\infty}$ (where $e_{m}$ denotes the element with 1 in the $m^{\text {th }}$ place and 0 elsewhere). Consider the sets 
$A=\left\{a e_{i}: 0<a \leq 1\right\}$ and $B=\left\{b e_{j}: 0<b \leq 1\right\}, \quad$ where $i \neq j$.

Let $T: A \cup B \longrightarrow A \cup B$ be a mapping defined by

$$
T\left(a e_{i}\right)=\sqrt{a} e_{j} \text { and } T\left(b e_{j}\right)=\sqrt{b} e_{i} .
$$

Clearly, $T$ is not a cyclic relatively nonexpansive mapping. Let $u=a e_{i}$ and $v=b e_{j}$. Then,

$$
\begin{aligned}
& \|T u-T v\|_{\infty}=\left\|\sqrt{a} e_{j}-\sqrt{b} e_{i}\right\|_{\infty}=\max \{\sqrt{a}, \sqrt{b}\}, \\
& R(u, O(v))=\sup _{n}\left\|u-T^{n} v\right\|_{\infty}=\sup _{n} \max \left\{a, \sqrt[2^{n}]{b}\right\}=1, \\
& R(v, O(u))=\sup _{n}\left\|v-T^{n} u\right\|_{\infty}=\sup _{n} \max \left\{b, \sqrt[2^{n}]{a}\right\}=1 .
\end{aligned}
$$

It shows that $T$ is a cyclic relatively nonexpansive mapping with respect to orbits.

Now, we prove the following proposition.

Proposition 2. If $T: G \cup H \longrightarrow G \cup H$ is a cyclic relatively nonexpansive mapping, then $T$ is a cyclic relatively nonexpansive mapping with respect to orbits.

Proof. Let $T$ be a cyclic relatively nonexpansive mapping. For $u \in G, v \in H,\|T u-T v\| \leq\|u-v\| \leq R(u, O(v))$. Similarly, $\|T v-T u\| \leq R(v, O(u))$. This shows that $T$ is a cyclic relatively nonexpansive mapping with respect to orbits.

Lemma 2. Let $X$ be a strictly convex Banach space and let $(C, D)$ be a nonempty, convex weakly compact proximal pair of subsets in $X$. Suppose $(C, D)$ has the rectangle property. Let $T: C \cup D \longrightarrow C \cup D$ be a cyclic relatively nonexpansive mapping with respect to orbits. Further, assume that proximal pair $(C, D)$ does not contain any proximal pair $(G, H)$ of subsets in $X$ such that $T(G) \subseteq H, T(H) \subseteq G$. Then, $(C, D) \subseteq(\overline{\operatorname{conv}}(T(D)), \overline{\operatorname{conv}}(T(C)))$.

Proof. Let $G=\overline{\operatorname{conv}}(T(D)) \cap C$ and $H=\overline{\operatorname{conv}}(T(C)) \cap D$. Then, $G$ and $H$ are, respectively, convex weakly compact subsets of $C$ and $D$. Suppose $(u, v) \in C \times D$ such that $\|u-v\|=$ $\operatorname{dist}(C, D)$. Then, $(T v, T u) \in T(D) \times T(C)$ and $(T(D)$, $T(C) \subseteq(C, D)$ which implies $(T v, T u) \in G \times H$. Since $\|u-v\|=\operatorname{dist}(C, D)$, by Definition 8 , we get $\| T v-$ $T u \|=\operatorname{dist}(C, D)$. Hence, $\operatorname{dist}(G, H)=\operatorname{dist}(C, D)$. It suffices to prove that $(G, H)$ is a proximal pair if for every $u \in G$, there is $v \in H$ such that

$$
\operatorname{dist}(C, D)=\|u-v\| .
$$

Let $u \in \operatorname{conv}(T(D)) \cap C$. Then, $u=\sum_{i=1}^{n} c_{i} T v_{i}$, where $v_{i} \in D, c_{i} \geq 0$ and $\sum_{i=1}^{n} c_{i}=1$. Since $(C, D)$ is a proximal pair, there is $v_{i}^{\prime} \in C$ such that

$$
\operatorname{dist}(C, D)=\left\|v_{i}^{\prime}-v_{i}\right\|, \quad i=1,2, \ldots, n .
$$

Then, $u^{\prime}=\sum_{i=1}^{n} c_{i} T v_{i}^{\prime} \in \operatorname{conv}(T(C))$ such that $\left\|u-u^{\prime}\right\|=$ $\operatorname{dist}(C, D)$ and $u^{\prime} \in H$. Thus, $(G, H) \subseteq(C, D)$ is a proximal pair (and hence proximal parallel pair). It is easy to see that $(G, H)$ is invariant under $T$, that is, $T(G) \subseteq H, T(H) \subseteq G$. This proves that $(C, D)=(G, H)$. Hence, $(C, D) \subseteq(\overline{\operatorname{conv}}(T(D))$, $\overline{\operatorname{conv}}(T(C)))$.

Theorem 3. Let $X$ be a strictly convex Banach space and let $(A, B)$ be a nonempty, convex weakly compact proximal pair in $X$. Let $T: A \cup B \longrightarrow A \cup B$ be a cyclic relatively nonexpansive mapping with respect to orbits. Suppose $(A, B)$ has proximal normal structure and the rectangle property. Then, there is $(u, v) \in A \times B$ such that $\|u-T u\|=\operatorname{dist}(A$, $B)=\|v-T v\|$.

Proof. Let $\mathfrak{F}$ be the set of all nonempty, convex weakly closed proximal pair of subsets $(C, D)$ of $(A, B)$ satisfying $T(C) \subseteq D, T(D) \subseteq C$ and $\operatorname{dist}(A, B)=\operatorname{dist}(C, D)$. Let $\|\prec\|$ be a relation defined on $\mathfrak{F}$ by $\left(C_{1}, D_{1}\right) \prec\left(C_{2}, D_{2}\right)$ if and only if $\left(C_{1}, D_{1}\right) \subseteq\left(C_{2}, D_{2}\right)$, where $\left(C_{1}, D_{1}\right),\left(C_{2}, D_{2}\right) \in \mathfrak{F}$. It is easy to see that $\mathfrak{F}$ is a partially ordered set. Also, if $\mathscr{T}$ is a totally ordered set, then $\mathscr{T} \subseteq \mathfrak{F}$ has the finite intersection property (FIP). Hence,

$$
(E, F):=\bigcap_{(C, D) \in \mathscr{T}}(C, D)
$$

is a nonempty, convex weakly compact proximal pair and $(E, F) \in \mathfrak{F}$. By Zorn's lemma, partially ordered set $\mathfrak{F}$ has a minimal element, say $(C, D)$. Therefore, from Lemma 2 , we conclude that

$$
(C, D) \subseteq(\overline{\operatorname{conv}}(T(D)), \overline{\operatorname{conv}}(T(C))) .
$$

If $\delta(C, D)=\operatorname{dist}(A, B)$, we get our result and the theorem is complete. Suppose

$$
\delta(C, D)>\operatorname{dist}(A, B) .
$$

By proximal normal structure, there is $\left(u_{1}, v_{1}\right) \in C \times D$ and $\eta \in] 0,1[$ such that

$$
\max \left\{R\left(u_{1}, D\right), R\left(v_{1}, C\right)\right\} \leq \eta \delta(C, D) .
$$

Since $(C, D)$ is a proximal pair, there is $\left(u_{1}^{\prime}, v_{1}^{\prime}\right) \in C \times D$ such that

$$
\left\|u_{1}-v_{1}^{\prime}\right\|=\left\|u_{1}^{\prime}-v_{1}\right\|=\operatorname{dist}(A, B) .
$$

So, for any $w \in D$, we have

$$
\begin{aligned}
\left\|\frac{u_{1}+u_{1}^{\prime}}{2}-w\right\| & \leq\left\|\frac{u_{1}-w}{2}\right\|+\left\|\frac{u_{1}^{\prime}-w \|}{2}\right\| \\
& \leq \frac{\eta \delta(C, D)}{2}+\frac{\delta(C, D)}{2} \\
& \left.\leq \beta \delta(C, D), \quad \text { where, } \beta=\frac{1+\eta}{2} \in\right] 0,1[.
\end{aligned}
$$

Let $u_{2}=\left(u_{1}+u_{1}^{\prime} / 2\right)$ and $v_{2}=\left(v_{1}+v_{1}^{\prime} / 2\right)$. Then,

$$
\begin{aligned}
R\left(u_{2}, D\right) & \leq \beta \delta(C, D), R\left(v_{2}, C\right) \leq \beta \delta(C, D) \text { and }\left\|u_{2}-v_{2}\right\| \\
& =\operatorname{dist}(C, D) .
\end{aligned}
$$


Define

$$
\begin{aligned}
& G=\{u \in C: R(u, D) \leq \beta \delta(C, D)\} \\
& H=\{v \in D: R(v, C) \leq \beta \delta(C, D)\} .
\end{aligned}
$$

Then, $(G, H)$ is a nonempty, convex weakly compact proximal pair of subset of $(C, D)$. By Lemma $1,(G, H)$ is also a proximal parallel pair of subset of $(C, D)$. Now, we need to claim that $T(G) \subseteq H, T(H) \subseteq G$. Since $(C, D) \subseteq(\overline{\operatorname{conv}}(T(D)), \overline{\operatorname{conv}}(T(C)))$ and $(A, B)$ has rectangle property, for $u \in G$,

$$
\begin{aligned}
R(T u, C) & =\sup \{\|T u-w\|: \quad w \in C\} \leq \sup \{\|T u-w\|: w \in \overline{\operatorname{conv}}(T(D))\} \\
& =\sup \{\|T u-T v\|: T v \in T(D)\} \leq \sup \{R(u, O(v)): v \in D\} \leq R(u, D) \leq \beta \delta(C, D) .
\end{aligned}
$$

This shows that $T(G) \subseteq H$. Similarly, for $v \in H$,

$$
\begin{aligned}
R(T v, D) & =\sup \{\|T v-z\|: z \in D\} \leq \sup \{\|T v-z\|: z \in \overline{\operatorname{conv}}(T(C))\} \\
& =\sup \{\|T v-T u\|: T u \in T(C)\} \leq \sup \{R(v, O(u)): u \in C\} \leq R(v, C) \leq \beta \delta(C, D) .
\end{aligned}
$$

This shows that $T(H) \subseteq G$. Therefore, $(G, H) \in \mathfrak{F}$. But $\delta(C, D)=\sup _{u \in G} R(u, D) \leq \beta \delta(C, D)<\delta(C, D)$, which is a contradiction. This shows that $C$ and $D$ are singleton sets. Therefore, there is $(u, v) \in(A, B)$ such that $\|u-T u\|=\operatorname{dist}(A, B)=\|v-T v\|$.

In Theorem 3, if $A=B$, we obtain the result of Harandi et al. (see Theorem 2.2 in [4]). The following example illustrates that the rectangle property in Theorem 3 cannot be dropped.

Example 2. Let $X=\left(\mathbb{R}^{2},\|\cdot\|\right)$, an Euclidean space, and let

$$
\begin{aligned}
& A=\{(u, 5-5 u): u \in[0,1]\} \\
& B=\{(u+1,5-5 u): u \in[0,1]\}
\end{aligned}
$$

Then, the pair $(A, B)$ with $B=A+h, h=(1,0)$ satisfies all the assumptions of Theorem 3 except the rectangle property. It is easy to see that the pair $(A, B)$ does not have the rectangle property by taking particular points $(1,0),(0,5) \in A \quad$ and $\quad(2,0),(1,5) \in B$. Let $T: A \cup B \longrightarrow A \cup B$ be a mapping as in Definition 8 . We need to claim that

$$
\|T u-T v\| \leq R(u, O(v)) \leq R(u, B), \quad(u, v) \in A \times B, O(v):=\left\{v, T v, \ldots, T^{n} v \operatorname{dot} s\right\}, T^{2 n+1} v \in A, T^{2 n} v \in B
$$

$$
\begin{aligned}
& T\left(u_{1}, v_{1}\right)=\left(u_{1},-\frac{v_{1}}{2}\right)+(1,0), \\
& T\left(u_{2}, v_{2}\right)=\left(u_{2},-\frac{v_{2}}{4}\right)-(1,0),
\end{aligned}
$$

$T^{2 n+1}(v)=(0,5) \in O(v) \quad$ for some $\quad v \in B$, then $R(u, B)<R(u, O(v))$, which is absurd. It happened because $(A, B)$ does not have the rectangle property.

The next example will illustrate our result, Theorem 3.

Example 3. Let $X=\left(\mathbb{R}^{2},\|\cdot\|\right)$, an Euclidean space, and let

$$
\begin{aligned}
& A=\left\{\left(-\frac{1}{2}, v\right):-\frac{1}{2} \leq v \leq \frac{1}{2}\right\} \\
& B=\left\{\left(\frac{1}{2}, v\right):-\frac{1}{2} \leq v \leq \frac{1}{2}\right\} .
\end{aligned}
$$

Then, the pair $(A, B)$ satisfies all the assumptions of Theorem 3, and $B=A+h$, where $h=(1,0)$. Let $T: A \cup B \longrightarrow A \cup B$ be a mapping defined by

where $\left(u_{1}, v_{1}\right) \in A,\left(u_{2}, v_{2}\right) \in B$. Clearly, $T$ is cyclic relatively nonexpansive with respect to orbits but not cyclic relatively nonexpansive. Then, $\exists((-(1 / 2), 0),((1 / 2), 0)) \in A \times B$ such that

$$
\left\|\left(-\frac{1}{2}, 0\right)-T\left(-\frac{1}{2}, 0\right)\right\|=1=\left\|\left(\frac{1}{2}, 0\right)-T\left(\frac{1}{2}, 0\right)\right\| .
$$

\section{Results for Noncyclic Relatively Nonexpansive Mappings with respect to Orbits}

Gabeleh et al. [5] proved the following interesting theorem. 
Theorem 4. Let $X$ be a strictly convex Banach space and let $(C, D)$ be a nonempty, convex weakly compact pair in $X$. Then, the pair $(C, D)$ has proximal normal structure iff for every noncyclic relatively nonexpansive mapping $T$ with respect to orbits, there is $(u, v) \in C \times D$ such that $T u=u, T v=$ $v$ and $\|u-v\|=\operatorname{dist}(C, D)$.

In Theorem 4 , if $A=B$, the result of Harandi et al. (see Theorem 2.2 in [4]) is obtained. Next, we show that Mann's iteration process (see $[7,17,18])$ converges to a fixed point if $X$ is a uniformly convex Banach space.

Theorem 5. Let $X$ be a uniformly convex Banach space and let $A$ and $B$ be nonempty, convex closed bounded subsets of $X$. Let $T: A \cup B \longrightarrow A \cup B$ be a noncyclic relatively nonexpansive mapping with respect to orbits. Let an initial point $u_{0} \in A$, and define a sequence

$$
\begin{aligned}
u_{n+1} & =\left(1-\xi_{n}\right) u_{n}+\xi_{n} T u_{n}, \quad \text { where } \theta<\xi_{n} \\
& <1-\theta, \theta \in] 0,1 / 2], n=0,1,2,3, \ldots
\end{aligned}
$$

Then, $\quad \lim _{n \longrightarrow+\infty}\left\|u_{n}-T u_{n}\right\|=0 . \quad$ Moreover, if $d\left(u_{n}, A_{0}\right) \longrightarrow 0, T$ is continuous, and $T(A)$ is subset of a compact set, then the limit point of the sequence $\left\{u_{n}\right\}$ is a fixed point for $T$.

Proof. Suppose $\operatorname{dist}(A, B)>0$. From Theorem 4, there is $v \in B$ such that $T v=v$. Since

$$
\begin{aligned}
\left\|u_{n+1}-v\right\| & \leq\left(1-\xi_{n}\right)\left\|u_{n}-v\right\|+\xi_{n}\left\|T u_{n}-T v\right\| \\
& \leq\left(1-\xi_{n}\right)\left\|u_{n}-v\right\|+\xi_{n} R\left(u_{n}, O(v)\right) \\
& =\left\|u_{n}-v\right\|,
\end{aligned}
$$

and $\left\{\left\|u_{n}-v\right\|\right\}$ is nonincreasing sequence, there is a positive real number $k>0$ so that $\lim _{n \longrightarrow+\infty}\left\|u_{n}-v\right\|=k$. Since $X$ is uniformly convex, then $\delta(\varepsilon)>0$ for $\varepsilon>0$ is a strictly increasing (and continuous) function. So, it is possible to choose $\varepsilon_{1}>0$ so small that

$$
\left(1-a \delta\left(\frac{\varepsilon}{k+\varepsilon_{1}}\right)\right)\left(k+\varepsilon_{1}\right)<k, \quad \text { where, } a>0 .
$$

Let us assume that there exists a subsequence $\left\{u_{n_{i}}\right\}$ of $\left\{u_{n}\right\}$ with $\left\|u_{n_{i}}-T u_{n_{i}}\right\| \geq \varepsilon>0$. Choose $i$, such that $\left\|u_{n_{i}}-v\right\| \leq k+\varepsilon_{1}$. From Definition 6, we have $\left\|T u_{n_{i}}-T v\right\| \leq R\left(u_{n_{i}}, O(v)\right)=\left\|u_{n_{i}}-v\right\| \leq k+\varepsilon_{1}$. Now,

$$
\begin{aligned}
\left\|v-u_{n_{i+1}}\right\| & =\left\|\left(1-\xi_{n}\right)\left(v-u_{n_{i}}\right)+\xi_{n}\left(T v-T u_{n_{i}}\right)\right\| \\
& \leq\left(1-2 \delta\left(\frac{\varepsilon}{k+\varepsilon_{1}}\right) \min \left\{\xi_{n}, 1-\xi_{n}\right\}\right)\left(k+\varepsilon_{1}\right) \\
& \leq\left(1-b \delta\left(\frac{\varepsilon}{k+\varepsilon_{1}}\right)\right)\left(k+\varepsilon_{1}\right), \quad \text { where, } 0<b \\
& \leq 2 \min \left\{\xi_{n}, 1-\xi_{n}\right\} .
\end{aligned}
$$

By choosing $\varepsilon_{1}>0$ so small, we get

$$
\left(1-b \delta\left(\frac{\varepsilon}{k+\varepsilon_{1}}\right)\right)\left(k+\varepsilon_{1}\right)<k
$$

which is a contradiction.

Hence, $\lim _{n \longrightarrow+\infty}\left\|u_{n}-T u_{n}\right\|=\lim _{n \longrightarrow+\infty}\left\|u_{n}-u_{n+1}\right\|=0$. Since $T(A)$ is a subset of a compact set, the sequence $\left\{u_{n}\right\}$ has a subsequence $\left\{u_{n_{i}}\right\}$ such that $\lim _{i \longrightarrow+\infty} u_{n_{i}}=u \in A$. As $d\left(u_{n}, A_{0}\right) \longrightarrow 0$, there is $w_{n} \in A_{0}$ such that

$$
\lim _{n \longrightarrow+\infty}\left\|u_{n}-w_{n}\right\|=0 \text {. }
$$

Hence, there exists a subsequence $\left\{w_{n_{i}}\right\}$ of $\left\{w_{n}\right\}$ in $A_{0}$ such that $\lim _{i \longrightarrow+\infty} w_{n_{i}}=u \in A_{0}$. Thus, there is $w \in B_{0}$ such that $\|u-w\|=\operatorname{dist}(A, B)$. Now,

$$
\begin{aligned}
\left\|u_{n_{i+1}}-T w\right\|= & \|\left\{\left(1-\xi_{n}\right) u_{n_{i}}+\xi_{n} T u_{n_{i}}\right\} \\
& -\left\{\left(1-\xi_{n}\right) T w+\xi_{n} T w\right\} \| \\
\leq & \left(1-\xi_{n}\right)\left\|u_{n_{i}}-T w\right\|
\end{aligned}
$$

Since $\|u-w\|=\operatorname{dist}(A, B)$, by Definition 6, we get $\|T u-T w\|=\operatorname{dist}(A, B)$. Therefore, from equation (35), when $i \longrightarrow+\infty$, we have $\|u-T w\| \leq \operatorname{dist}(A, B)$ which implies $\|u-T w\|=\operatorname{dist}(A, B)$. By strict convexity of the norm, $T w=w$ which implies $T u=u$ because $u$ is the unique point of $A$ nearest to $w$.

From the above theorem, if $\operatorname{dist}(A, B)=0$, we get the following corollary.

Corollary 1. Let $X$ be a uniformly convex Banach space and let $A$ be nonempty, closed bounded convex subset of $X$. Let $T: A \longrightarrow A$ be a nonexpansive mapping with respect to orbits. Let the initial point $u_{0} \in A$, and define a sequence

$$
\begin{aligned}
u_{n+1} & =\left(1-\xi_{n}\right) u_{n}+\xi_{n} T u_{n}, \quad \text { where, } \theta<\xi_{n} \\
& <1-\theta, \theta \in] 0,1 / 2], n=0,1,2,3, \ldots
\end{aligned}
$$

Then, $\lim _{n \longrightarrow+\infty}\left\|u_{n}-T u_{n}\right\|=0$. Moreover, if $T$ is continuous and $T(A)$ is subset of a compact set, then the limit point of the sequence $\left\{u_{n}\right\}$ is a fixed point for $T$.

The following example illustrates Theorem 5 .

Example 4. Let $X=\left(\mathbb{R}^{2},\|\|.\right)$, an Euclidean space, and let

$$
\begin{aligned}
& A=\left\{(u, v):-2 \leq u \leq-1, \frac{-1}{2} \leq v \leq \frac{1}{2}\right\} \\
& B=\left\{(u, v): 1 \leq u \leq 2, \frac{-1}{2} \leq v \leq \frac{1}{2}\right\} .
\end{aligned}
$$

Define $T: A \longrightarrow A$ by

$$
T(u, v)=\left(T_{1} u, T_{2} v\right)=\left(\frac{u-6}{7},-\frac{v}{2}\right), \quad \text { for }(u, v) \in A,
$$


and $T: B \longrightarrow B$ by

$$
T(u, v)=\left(\frac{u+6}{7},-\frac{v}{4}\right), \text { for } \quad(u, v) \in B
$$

where

$$
T_{1}:[-2,-1] \longrightarrow[-2,-1]
$$

and

$T_{2}:[-(1 / 2),(1 / 2)] \longrightarrow[-(1 / 2),(1 / 2)]$. It clearly shows that $T: A \cup B \longrightarrow A \cup B$ is noncyclic relatively nonexpansive with respect to orbits but not noncyclic relatively nonexpansive. Let the initial point $(u, v) \in A$, and set $u_{1}=\left(1-\xi_{1}\right) u+\xi_{1} T_{1} u$. We have $T_{1} u=((u-6) / 7)$. Therefore,

$$
u_{1}=\frac{u\left(7-6 \xi_{1}\right)-6 \xi_{1}}{7}
$$

Following the same line of Example 2.2 in [7], we get

$$
\begin{aligned}
u_{n}= & \frac{1}{7^{n}}\left\{u\left(7-6 \xi_{1}\right) \cdots\left(7-6 \xi_{n}\right)-6 \xi_{1}\left(7-6 \xi_{2}\right) \cdots\left(7-6 \xi_{n}\right)\right. \\
& -6.7 \xi_{2}\left(7-6 \xi_{3}\right) \cdots\left(7-6 \xi_{n}\right) \\
\left.-\cdots-6.7^{n-2} \xi_{n-1}\left(7-6 \xi_{n}\right)-6.7^{n-1} \xi_{n}\right\}, &
\end{aligned}
$$

and $\lim _{n \longrightarrow \infty} u_{n}=-1$. Again, we have $T_{2} v=-(v / 2)$. Let

$$
v_{1}=\left(1-\xi_{1}\right) v+\xi_{1} T_{2} v=\frac{v\left(2-3 \xi_{1}\right)}{2} \text {. }
$$

Now, $T_{2} v_{1}=-\left(v_{1} / 2\right)=-\left(v\left(2-3 \xi_{1}\right) / 2^{2}\right)$. Therefore, $v_{2}=\left(1-\xi_{2}\right) v_{1}+\xi_{2} T_{2} v_{1}=\frac{v\left(2-3 \xi_{2}\right)\left(2-3 \xi_{1}\right)}{2^{2}}$, and so on.

In general,

$$
v_{n}=\frac{v\left(2-3 \xi_{n}\right)\left(2-3 \xi_{n-1}\right) \ldots\left(2-3 \xi_{1}\right)}{2^{n}},
$$

and $\quad \lim _{n \longrightarrow+\infty} v_{n}=0$. Hence, $\lim _{n \longrightarrow+\infty}\left(u_{n}, v_{n}\right)=$ $(-1,0) \in A$. Since $(-1,0) \in A_{0}$, there exists $(1,0) \in B_{0}$ such that $\|(-1,0)-(1,0)\|=2=\operatorname{dist}(A, B)$. Again, $\| T(-1,0)-$ $T(1,0) \|=2=\operatorname{dist}(A, B)$. This shows that sequence $\left(u_{n}, v_{n}\right) \longrightarrow(-1,0) \in A$ as $n \longrightarrow+\infty$, a fixed point for $T$. In a similar way, we can show that if $(u \prime, v \prime) \in B$, then $\lim _{n \longrightarrow+\infty}\left(u_{n}^{\prime}, v_{n}^{\prime}\right)=(1,0) \in B$, a fixed point for $T$.

\section{Conclusions}

From the results obtained in this article, we draw the following conclusions.

In this research article, we study the existence of the best proximity point for cyclic relatively nonexpansive mappings with respect to orbits. Additionally, this research article establishes the convergence of Mann's iteration process for a noncyclic relatively nonexpansive mapping with respect to orbits and nonexpansive mapping with respect to orbits to a fixed point. There may be scope for extension of results obtained in Section 3 by using other well-known iteration processes.

\section{Data Availability}

No data were used in this study.

\section{Conflicts of Interest}

The authors declare that they have no conflicts of interest.

\section{Acknowledgments}

Laishram Shanjit was financially supported by the University Grant Commission, India, fellowship grant no. 420004.

\section{References}

[1] M. S. Brodskiĭ and D. P. Milman, "On the center of a convex set," Doklady Akademii Nauk SSSR (N. S.), vol. 59, pp. 837840, 1948, in Russian.

[2] W. A. Kirk, "A fixed point theorem for mappings which do not increase distances," The American Mathematical Monthly, vol. 72, no. 9, pp. 1004-1006, 1965.

[3] A. A. Eldred, W. A. Kirk, and P. Veeramani, "Proximal normal structure and relatively nonexpansive mappings," Studia Mathematica, vol. 171, no. 3, pp. 283-293, 2005.

[4] A. A. Harandi, M. Fakhar, and H. R. Hajisharifi, "Weak fixed point property for nonexpansive mappings with respect to orbits in Banach spaces," Journal of Fixed Point Theory and Applications, vol. 18, pp. 601-607, 2016.

[5] M. Gabeleh and S. P. Moshokoa, "Study of minimal pairs for relatively nonexpansive mappings with respect to orbits," Optimization, pp. 1-17, 2020.

[6] D. E. Alspach, "A fixed point free nonexpansive map," Proceedings of the American Mathematical Society, vol. 82, no. 3, pp. 423-424, 1981.

[7] A. A. Eldred, A. Praveen, and A. Praveen, "Convergence of Mann's iteration for relatively nonexpansive mappings," Fixed Point Theory, vol. 18, no. 2, pp. 545-554, 2017.

[8] E. L. Fuster, "Normal structure and orbital fixed point conditions," Journal of Mathematical Analysis and Applications, vol. 72, no. 2, pp. 1004-1006, 2018.

[9] A. Hussain, M. Adeel, T. Kanwal, and N. Sultana, "Set valued contraction of Suzuki-Edelstein-Wardowski type and best proximity point results," Bulletin of Mathematical Analysis and Applications, vol. 10, no. 2, pp. 53-67, 2018, http:// bmathaa.org.

[10] Z. D. Mitrovic, "Optimal solutions and applications to nonlinear matrix and integral equations via Simulation function," Filomat, vol. 32, no. 17, pp. 6087-6106, 2018, http:// journal.pmf.ni.ac.rs/filomat/index.php/filomat/article/view/ 8245.

[11] M. Abbas, V. Rakocevic, V. Rakočević, and A. Hussain, "Best proximity point of Zamfirescu contractions of Perov type on 
regular cone metric spaces," Fixed Point Theory, vol. 21, no. 1, pp. 3-18, 2020.

[12] A. Hussain, M. Abbas, M. Adeel, and T. Kanwal, "Best proximity point results of multivalued almost contraction mappings and application to nonlinear differential equation," Sahand Communications in Mathematical Analysis, vol. 17, no. 2, pp. 119-138, 2020.

[13] R. Espínola, "A new approach to relatively nonexpansive mappings," Proceedings of the American Mathematical Society, vol. 136, no. 06, pp. 1987-1995, 2008.

[14] S. Rajesh and P. Veeramani, "Best proximity point theorems for asymptotically relatively nonexpansive mappings," $\mathrm{Nu}$ merical Functional Analysis and Optimization, vol. 37, no. 1, pp. 80-91, 2016.

[15] J. A. Clarkson, "Uniformly convex spaces," Transactions of the American Mathematical Society, vol. 40, no. 3, pp. 396-414, 1936, https://www.jstor.org/stable/1989630.

[16] C. E. Chidume, Geometric Properties of Banach Spaces and Nonlinear Iterations, Springer, London, UK, 2009, https:// www.springer.com/gp/book/9781848821897.

[17] W. R. Mann, "Mean value methods in iteration," Proceedings of the American Mathematical Society, vol. 4, no. 3, pp. 506-510, 1953.

[18] H. F. Senter and W. G. Dotson, "Approximating fixed points of nonexpansive mappings," Proceedings of the American Mathematical Society, vol. 44, no. 2, pp. 375-380, 1974, https://www.jstor.org/stable/2040440. 